\title{
Compact Kähler 3-manifolds without nontrivial subvarieties
}

\author{
Frédéric Campana, Jean-Pierre Demailly and Misha Verbitsky
}

\begin{abstract}
We prove that any compact Kähler 3-dimensional manifold which has no nontrivial complex subvarieties is a torus. This is a very special case of a general conjecture on the structure of so-called simple manifolds, central in the bimeromorphic classification of compact Kähler manifolds. The proof follows from the Brunella pseudo-effectivity theorem, combined with fundamental results of Siu and of the second author on the Lelong numbers of closed positive $(1,1)$-currents, and with a version of the hard Lefschetz theorem for pseudo-effective line bundles, due to Takegoshi and Demailly-PeternellSchneider. In a similar vein, we show that a normal compact and Kähler 3-dimensional analytic space with terminal singularities and nef canonical bundle is a cyclic quotient of a simple nonprojective torus if it carries no effective divisor. This is a crucial step towards completing the bimeromorphic classification of compact Kähler threefolds.
\end{abstract}

\section{Introduction}

We consider here connected compact Kähler manifolds $X$ of (complex) dimension $n>1$. An irreducible compact analytic subset $Z$ of $X$ will be said to be a subvariety of $X$. It is said to be nontrivial if its (complex) dimension is neither 0 nor $n$.

The bimeromorphic classification of compact Kähler manifolds can be reduced, by means of suitable functorial fibrations, to the following two extreme particular cases: either $X$ is projective or $X$ is simple, which means that any general point $x$ of $X$ is not contained in any nontrivial subvariety of $X$ (a point is general if it lies in the intersection of countably many dense Zariski open subsets). If $X$ is a nonprojective torus, this new notion of simpleness coincides with the classical one, defined by the absence of nontrivial subtori. The functorial fibrations needed for this reduction are relative algebraic reductions and relative Albanese maps (see [Cam80], [Cam85], [Fuj83]) in the category of connected compact Kähler manifolds, where the morphisms are the dominant rational maps with connected fibre.

The present text is concerned with the bimeromorphic classification of such simple $X$. Its difficulty, in contrast to the projective case, is not due to the abundance and complexity of the

Received 29 April 2013, accepted in final form 4 September 2013.

2010 Mathematics Subject Classification 32J17, 32J25, 32J27

Keywords: Kähler manifold, complex threefold, holomorpic foliation, nef bundle, pseudoeffective bundle, hard Lefschetz theorem, Lelong number

This journal is (C) Foundation Compositio Mathematica 2014. This article is distributed with Open Access under the terms of the Creative Commons Attribution Non-Commercial License, which permits non-commercial reuse, distribution, and reproduction in any medium, provided that the original work is properly cited. For commercial re-use, please contact the Foundation Compositio Mathematica.

Misha Verbitsky is partially supported by RFBR grants 12-01-00944-a, AG Laboratory NRI-HSE, RF government grant, ag. 11.G34.31.0023, and NRI-HSE Academic Fund Program in 2013-2014, research grant 12-01-0179. 


\section{F .Campana, J.-P. Demailly and M. Verbitsky}

examples, but to their (expected) scarceness and simple structure, which makes essentially all usual invariants of the classification vanish.

For example, if $X$ is simple, its algebraic dimension ${ }^{1} a(X)$ vanishes, which implies, by [Uen75], Thm. 9.3, that its Albanese map is surjective and has connected fibres. Because the fibres must then have dimension either 0 or $n$, we get: either $q=0$ or $X$ is bimeromorphic to its Albanese torus. Thus only the case where $q=0$ needs to be considered.

The known ${ }^{2}$ examples of simple compact Kähler manifolds are (up to bimeromorphic equivalence) general complex tori, $K 3$ surfaces or the general member of the deformation families of hyperkähler manifolds ${ }^{3}$. Simple surfaces are thus classified: they are either tori $(q>0)$ or $K 3(\mathrm{q}=0)$. But when $n \geqslant 3$, the classification is open. The situation is however expected to be similar to the surface case in higher dimensions (see Conjecture 1.1), where the only known examples are constructed out of surfaces which are either $K 3$ or tori.

The following converse was essentially formulated in [Cam06], as question 1.4.

Conjecture 1.1. Let $X$ be a simple compact Kähler manifold. Then the following hold.

1. Either $X$ has a finite étale cover bimeromorphic to a complex torus or $H^{0}\left(X, \Omega_{X}^{2}\right)$ is generated by some $\sigma$ which is generically symplectic (that is, $n=2 m$ is even and $\wedge^{m} \sigma \neq 0$ ).

We should thus have: $\kappa(X)=0$. If $\operatorname{dim}(X)$ is odd, then $X$ should be bimeromorphic to a complex torus, possibly after some finite étale cover. Such a cover will be needed, as shown by the Kummer quotient by the -1 involution.

2. When $X$ does not contain any nontrivial subvarieties, $X$ should be either a complex torus or an irreducible hyperkähler manifold ${ }^{4}$.

Thus $K_{X}$ should be trivial in this case, the two cases being distinguished by $q>0$ or $q=0$.

3. If $X$ is generically symplectic, then $\pi_{1}(X)$ should be finite, of cardinality at most $2^{m}$, where $2 m=n$.

Assertion 3 follows from [Cam85] if $\chi\left(\mathcal{O}_{X}\right) \neq 0$, for $X$ simple and generically symplectic. In what follows, we prove the second assertion of the conjecture for $n=3$.

This conjecture can be motivated by the conjectural existence of minimal models in the bimeromorphic category of connected compact Kähler manifolds. Indeed, if such a theory exists, and if $X$ is simple, we have $a(X)=0$, which brings $\kappa(X) \leqslant 0$. The possibility $\kappa(X)=-\infty$ is excluded, since $X$ should then be uniruled. This implies that $\kappa(X)=0$.

In this case, a Kähler minimal model is a map $X \rightarrow X^{\prime}$ such that $X^{\prime}$ has terminal singularities and $K_{X^{\prime}} \equiv 0$. A (conjectural) Bogomolov-type decomposition ([Bog74], [Bea83]) for manifolds with terminal singularities and $K_{X^{\prime}} \equiv 0$ could be used to represent a finite cover of $X^{\prime}$ as a product. However, $X$ is simple, hence either $X^{\prime}$ is covered by a simple torus (with finite locus of ramification) or $X^{\prime}$ carries a symplectic holomorphic 2 -form, establishing the conjecture above.

\footnotetext{
${ }^{1}$ Recall that $a(X) \in\{0, \ldots, n\}$ is the transcendence degree of the field of global meromorphic functions on $X$, $a(X)=n$ if and only if $X$ is projective.

${ }^{2}$ It is shown in [KV01] that a stable bundle which does not degenerate to a direct sum of stable bundles on the generic deformation of a Hilbert scheme of $K 3$ has a compact moduli space; this may lead to new examples of hyperkähler manifolds. The rigidity of the category of coherent sheaves on the general members of these families is established in [Ver08], [Ver04].

${ }^{3}$ Recall that $X$ is hyperkähler it is Kähler and admits a holomorphic symplectic form. It is said to be irreducible if, moreover, $\pi_{1}(X)$ is finite, and $h^{2,0}(X)=1$.

${ }^{4}$ Notice that a general deformation of a Hilbert scheme of a $K 3$ surface has no subvarieties [Ver98], while a general deformation of a generalized Kummer variety has some subvarieties, partially classified in [KV98].
} 


\section{KÄHLER THREEFOLDS HAVING NO SUBVARIETIES}

The above conjecture can thus be reformulated in terms of conjectural minimal models for compact Kähler manifolds, defined as normal compact complex spaces bimeromorphic to compact Kähler manifolds, and having $\mathbb{Q}$-factorial terminal singularities. The simple ones should then have a torsion canonical sheaf and be either of the form $T / G$, quotients of simple nonprojective tori by a finite group of automorphisms or carry a multiplicatively unique holomorphic 2-form, symplectic on the regular locus.

Theorem 1.2. Let $X$ be a compact Kähler 3-dimensional manifold. Assume that $X$ has no nontrivial subvariety. Then $X$ is a torus.

The proof will be given as Corollary 4.4. Although it is an easy combination of known results, it seems worthwhile to present it, because it indicates that the above conjecture might be accessible in dimension 3 with the actually existing techniques. The proof also gives some information on the higher dimensional case (see Corollary 4.5).

We also show the following result.

Theorem 1.3. Let $X$ be a 3-dimensional Kähler normal connected compact complex space with terminal singularities. Assume that $K_{X}$ is nef, and that $X$ does not contain any effective divisors ${ }^{5}$. Then $X=T / G$ is isomorphic to the quotient of a simple torus by a finite cyclic group of automorphisms.

We will use the following simple criterion.

Lemma 1.4. Let $X$ be a simple compact Kähler threefold. If $\chi\left(\mathcal{O}_{X}\right)=0$, then $X$ is bimeromorphic to its Albanese torus.

Proof. By the above remarks, it is sufficient to show that $q>0$. But

$$
0=\chi\left(\mathcal{O}_{X}\right)=1-q+h^{2,0}-h^{3,0} .
$$

By Kodaira's theorem, $h^{2,0}>0$ since $X$ is not projective and $h^{3,0} \leqslant 1$ since $a(X)=0$. Thus $0=1-q+h^{2,0}-h^{3,0} \geqslant 1-q+1-1=1-q$ and $q>0$.

The proof of Theorem 1.2 is thus reduced to showing that $\chi\left(\mathcal{O}_{X}\right)=0$, which is the objective of the following sections.

Finally, let us mention that compact (connected) complex manifolds, not necessarily Kähler, without nontrivial subvarieties are also quite important in model theory, giving examples of socalled trivial Zariski geometries ([Moo05], [MP08] [MMT08]). Our arguments below show that compact 3-dimensional complex manifolds without nontrivial subvarieties are tori, provided their canonical bundle are pseudo-effective. Brunella's theorem and the hard Lefschetz theorem appear to be the only steps of the proof requiring the Kähler hypothesis in depth.

\section{Pseudoeffective line bundles}

We refer to [Dem07] for the basic notions and properties of currents on complex manifolds and only recall briefly some of the facts needed here.

Let $X$ be a compact, $n$-dimensional complex manifold and let $L$ be a holomorphic line bundle on $X$. Assume that $L$ is equipped with a singular hermitian metric $h$ with local weights $e^{-\varphi}$

\footnotetext{
${ }^{5}$ The nonexistence of effective divisors linearly equivalent to a positive multiple of $K_{X}$ actually suffices for the conclusion.
} 


\section{F .Campana, J.-P. Demailly and M. Verbitsky}

which are locally integrable. Its curvature current is then $\Theta(L, h):=i \partial \bar{\partial} \varphi$ and its first Chern class $c_{1}(L)$ is represented by the cohomology class $\frac{1}{2 \pi}[\Theta(L, h)]$ in the Bott-Chern cohomology group $H_{\mathrm{BC}}^{1,1}(X)$, where

$$
H_{\mathrm{BC}}^{p, q}(X)=\{d \text {-closed }(p, q) \text {-currents }\} /\{\partial \bar{\partial} \text {-exact }(p, q) \text {-currents }\} \text {. }
$$

If $X$ is Kähler, $H_{\mathrm{BC}}^{1,1}(X)$ is isomorphic to the Dolbeault cohomology group $H^{1,1}(X)$ and can be viewed as a subspace of $H^{2}(X, \mathbb{R})$.

Definition 2.1. A $(1,1)$ cohomology class $\{\alpha\} \in H_{\mathrm{BC}}^{1,1}(X)$ is said to be pseudo-effective if it contains a closed positive $(1,1)$-current $\Theta$. The class $\{\alpha\}$ is said to be nef if for a given smooth positive $(1,1)$-form $\omega>0$ on $X$ and every $\varepsilon>0$, it contains a smooth closed $(1,1)$-form $\alpha_{\varepsilon}$ such that $\alpha_{\varepsilon} \geqslant-\varepsilon \omega$ (or alternatively, in the Kähler case, if $\{\alpha\}$ is a limit of Kähler classes $\{\alpha+\varepsilon \omega\}$, where $\omega$ is Kähler). A class $\{\alpha\}$ is said to be big if it contains a Kähler current, namely a closed $(1,1)$-current $\Theta$ such that $\Theta \geqslant \varepsilon \omega$ for some smooth positive $(1,1)$-form $\omega$ and some $\varepsilon>0$. A line bundle $L$ on $X$ is said to be nef (resp. pseudo-effective, resp. big) if its first Chern class $c_{1}(L) \in H_{\mathrm{BC}}^{1,1}(X)$ has the same property.

It follows from the Bochner-Kodaira technique that a big line bundle $L \rightarrow X$ has maximal Kodaira dimension $\kappa(L)=\operatorname{dim} X$. Therefore a compact complex manifold $X$ carrying a big line bundle is Moishezon, that is, bimeromorphic to a projective algebraic manifold; in particular, $X$ is not simple. Now, we have the following result.

Theorem 2.2. ([DPa04]) Let $X$ be a compact Kähler manifold. Let $\{\alpha\} \in H^{1,1}(X)$ be a nef class. If $\int_{X} \alpha^{n}>0$, then $\{\alpha\}$ is big. As a consequence, if $X$ carries a nef line bundle $L$ such that $c_{1}(L)^{n}>0$, then $L$ is big and $X$ cannot be simple.

This special case where $\{\alpha\}=c_{1}(L)$ (the only one that we need here) can also be obtained as a consequence of holomorphic Morse inequalities [Dem91]. It can be seen as a strengthening of the Grauert-Riemenschneider conjecture proved by Siu [Siu84] (although the latter is also valid in the non-Kähler case).

Definition 2.3. The Lelong number $\nu_{x}(\Theta(L, h))=\nu(\varphi, x)$ of the $(1,1)$-current $\Theta(L, h)$ at $x \in X$ is defined as $\liminf z \rightarrow x \frac{\varphi(z)}{|z-x|}$ for a local metric on $X$ near $x$.

Definition 2.4. For a positive real number $c>0$, the Lelong set $F_{c}$ of a $(1,1)$-current $\eta$ is the set of points $x \in M$ with $\nu(\eta, x) \geqslant c$. By a well-known theorem of Siu ([Siu74]), any Lelong set of a positive, closed current is a complex analytic subvariety of $M$ (the proof of this difficult result has been considerably simplified, using regularization of currents and the Ohsawa-Takegoshi extension theorem, see [Dem92].)

Theorem 2.5. ([Dem92]) Let $X$ be a compact complex manifold. Let $L$ be a pseudo-effective holomorphic line bundle on $X$, with singular hermitian metric $h$ with positive curvature current $\Theta(L, h)$. Assume that the Lelong sets of $\Theta(L, h)$ are all zero-dimensional. Then $L$ is nef, and big as soon as there is at least one nonzero Lelong number.

Proof. The first assertion is ([Dem92], Cor. 6.4). The second follows from ([Dem92], Cor. 7.6). See also [Ver09], Thm. 3.12.

Corollary 2.6. If $X$ is a compact Kähler manifold without nontrivial subvarieties and with pseudo-effective canonical bundle, then $K_{X}$ is nef and for any singular hermitian metric $h$ on 


\section{KÄHLER THREEFOLDS HAVING NO SUBVARIETIES}

$K_{X}^{\otimes m}, m>0$, with positive curvature current, the Lelong number vanishes at any point. In particular, the associated multiplier ideal sheaves on $K_{X}^{\otimes m}$ are all trivial for any $m>0$ (that is, $e^{-m \varphi}$ is integrable for any $m>0$ ).

\section{Hard Lefschetz theorem for the cohomology of pseudo-effective line bundles}

We recall the version of the Hard Lefschetz theorem which is going to be used here.

Theorem 3.1 ([Tak97], [DPS01]). Let $(X, \omega)$ be a compact Kähler manifold, of dimension $n$ with Kähler form $\omega$, let $K_{X}$ be its canonical bundle, and let $L$ be a pseudo-effective holomorphic line bundle on $X$ equipped with a singular Hermitian metric $h$. Assume that the curvature $\Theta$ of $(L, h)$ is a positive current on $X$ and denote by $\mathcal{I}(h)$ the corresponding multiplier ideal sheaf. Then the wedge multiplication operator $\eta \longrightarrow \omega^{i} \wedge \eta$ induces a surjective map

$$
H^{0}\left(X, \Omega_{X}^{n-i} \otimes L \otimes \mathcal{I}(h)\right) \stackrel{\omega^{i} \wedge}{\longrightarrow} H^{i}(X, K \otimes L \otimes \mathcal{I}(h)) .
$$

Here $\omega$ is considered as an element in $H^{1}\left(X, \Omega_{X}^{1}\right)$ and multiplication by $\omega$ maps $H^{k}\left(X, \Omega_{X}^{n-l} \otimes F\right)$ to $H^{k+1}\left(X, \Omega_{X}^{n-l+1} \otimes F\right)$.

This theorem was obtained under various hypotheses during the 1990s, see [Eno93], and [Mou99]. The most general form given here is due to [DPS01], Thm. 2.1.1. It was proved in [Tak97] when $L$ nef.

Corollary 3.2. Let $X$ be a compact Kähler manifold of dimension $n>1$ without any nontrivial subvarieties. Assume that $K_{X}$ is pseudo-effective. Then $h^{i}\left(X, m . K_{X}\right) \leqslant\left(\begin{array}{c}n \\ i\end{array}\right)$, for any $i \geqslant 0$, and the polynomial $P(m):=\chi\left(X, m . K_{X}\right)$ is constant, equal to $\chi\left(\mathcal{O}_{X}\right)$.

Proof. Since $n>1$ and $X$ has no nontrivial subvarieties, the algebraic dimension of $X$ vanishes, $a(X)=0$. Therefore $h^{0}(X, E) \leqslant \operatorname{rank}(E)$ for any holomorphic vector bundle $E$ on $X$. Combined with the Hard Lefschetz theorem (3.1), this gives the first claim of the corollary. The second claim is clear because a polynomial function $P(m)$ which remains bounded when $m \rightarrow+\infty$ is necessarily constant.

Corollary 3.3. Let $X$ be a compact Kähler manifold of dimension 3 without any nontrivial subvarieties. Assume that $K_{X}$ is pseudo-effective. The polynomial $P(m):=\chi\left(X, m . K_{X}\right)$ is constant, equal to $\chi\left(\mathcal{O}_{X}\right)=0$.

Proof. The intersection number $K_{X}^{3}$ vanishes (either by Theorem 2.5 or because it is the leading term of $P(m)$, up to the factor 3!). The Riemann-Roch formula therefore gives $P(m)=$ $\frac{(1-12 m)}{24} \cdot c_{1}(X) \cdot c_{2}(X)$. The boundedness of $P(m)$ then implies that 24. $\chi\left(\mathcal{O}_{X}\right)=c_{1}(X) \cdot c_{2}(X)=0$.

Remark 3.4. Arguments close to some of the ones presented here have already been used in the proof [DPS01], Thm. 2.7.3: if $X$ is a compact Kähler manifold with pseudo-effective canonical bundle admitting a metric with weights $\varphi$ having analytic singularities and positive curvature current, then either $H^{0}\left(X, \Omega_{X}^{i} \otimes\left(m . K_{X}\right)\right) \neq 0$ for infinitely many $m>0$ and some $i \geqslant 0$, or $\chi\left(X, \mathcal{O}_{X}\right)=0$.

\section{Brunella's pseudo-effectivity criterion.}

The rest of our arguments is based on the following strong (and very difficult) theorem by Brunella. 


\section{F .Campana, J.-P. Demailly and M. Verbitsky}

Theorem 4.1. ([Bru98]) Let $X$ be a compact Kähler manifold with a 1-dimensional holomorphic foliation $F$ given by a nonzero morphism of vector bundle $L \rightarrow T_{X}$, where $L$ is a line bundle on $X$ and $T_{X}$ is its holomorphic tangent bundle. If $L^{-1}$ is not pseudo-effective, then the closures of the leaves of $F$ are rational curves (and $X$ is thus uniruled).

The following corollary had already been observed in [HPR11], Prop. 4.2.

Corollary 4.2. If $X$ is an $n$-dimensional compact Kähler manifold with $H^{0}\left(X, \Omega_{X}^{n-1}\right) \neq 0$, then $K_{X}$ is pseudo-effective if $X$ is not uniruled.

Proof. The vector bundle $\Omega_{X}^{n-1}$ is canonically isomorphic to $K_{X} \otimes T_{X}$. Any nonzero section thus provides a nonzero map $K_{X}^{-1} \rightarrow T_{X}$ and an associated foliation

Corollary 4.3. If $X$ is a 3-dimensional nonprojective compact Kähler manifold, then either $K_{X}$ is pseudo-effective or $X$ is uniruled. If $X$ is simple, then $K_{X}$ is pseudo-effective.

Proof. Kodaira's theorem implies that any compact Kähler manifold with $H^{2,0}(X)=0$ is projective. Thus $H^{2,0}(X) \neq 0$, and the preceding corollary applies (since $2=(n-1)$, here) and gives the first claim. If $X$ is uniruled, it is not simple, whence the second assertion.

By combining Corollary 4.3, Corollary 3.3, and Lemma 1.4, we get our main result:

Corollary 4.4. If $X$ is a 3-dimensional compact Kähler manifold without nontrivial subvarieties, then $X$ is a complex torus.

In higher dimensions we can replace the assumption from Corollary 3.2 that $K_{X}$ was pseudoeffective with the existence of a holomorphic $(n-1)$-form, giving the following result.

Corollary 4.5. Let $X$ be a compact Kähler manifold of dimension $n>1$ without nontrivial subvarieties. Assume that $H^{0}\left(X, \Omega_{X}^{n-1}\right) \neq 0$. Then $h^{i}\left(X, m . K_{X}\right) \leqslant\left(\begin{array}{c}n \\ i\end{array}\right)$ for any $i \geqslant 0$ and the polynomial $P(m):=\chi\left(X, m . K_{X}\right)$ is constant, equal to $\chi\left(\mathcal{O}_{X}\right)$. (The intersection numbers $K_{X}^{j} \cdot \operatorname{Todd}_{n-j}(X)$ thus all vanish when $j>0$, as expected since $K_{X}$ should then be trivial.)

The following result (announced as Theorem 1.3) strengthens Theorem 1.2.

Theorem 4.6. Let $X$ be a 3-dimensional Kähler normal connected compact complex space with terminal ${ }^{6}$ singularities. Assume that $K_{X}$ is nef and that $X$ does not contain any effective divisors $^{7}$. Then $X=T / G$ is isomorphic to the quotient of a nonprojective simple torus by a finite cyclic group of automorphisms.

Proof. By [DPe03], Thm. 7.1, $\kappa(X)=0$. Thus $m K_{X}$ is a trivial line bundle for some $m>0$. Let $X^{\prime} \rightarrow X$ be the cyclic cover of $X$ defined by a nonzero section of $m . K_{X}$ so that $m . K_{X}$ is Cartier. The singularities of $X^{\prime}$ are thus terminal as well ([KM98], 5.20). They are also rational, since $K_{X^{\prime}}$ is Cartier (by [KM98], 5.24). We thus have $\chi\left(\mathcal{O}_{X^{\prime}}\right)=\chi\left(\mathcal{O}_{X^{\prime \prime}}\right)$ for any smooth model $X^{\prime \prime}$ of $X^{\prime}$. But

$$
\chi\left(\mathcal{O}_{X^{\prime}}\right)=\frac{-K_{X^{\prime}} \cdot c_{2}\left(X^{\prime}\right)}{24}=0,
$$

since $K_{X^{\prime}}$ is locally free trivial (see for example [Fle87]). The conclusion now follows from Lemma 1.4

${ }^{6}$ We refer to [KM98] for this notion, which makes sense without change in the analytic category. In particular, the sheaf $K_{X}$ is then $\mathbb{Q}$-Cartier.

${ }^{7}$ Divisors linearly equivalent to a positive multiple of $K_{X}$ actually suffice. This second hypothesis should follow from the first if $X$ is simple. 


\section{KÄHLER THREEFOLDS HAVING NO SUBVARIETIES}

Remark 4.7. In order to show the conjecture for simple Kähler threefolds, it would be sufficient

(i) to show the following statement (which is a form of the abundance conjecture in this context): If $X$ is a simple (normal, terminal, $\mathbb{Q}$-factorial, Kähler) threefold and $D$ is an effective divisor on $X$, then $D$ is not nef. A possible approach might consist in adapting the proof in the projective case. A more direct approach is however desirable.

(ii) to show the existence of minimal models such as the ones appearing in Theorem 1.3 for any compact Kähler threefold $X$. (This step has just been achieved in [HP13], where a conjecture equivalent to the one above is formulated in dimension 3.)

Remark 4.8. In [BGL99], it is shown that the only group $G$ that can act on a 3-dimensional torus without nontrivial analytic subvarieties is $\mathbb{Z}_{2}$ acting by \pm Id. In fact, from their classification (cf. the proof of Th. 4.1 and Prop. 3.6 in [BGL99]), any other nontrivial action corresponds to a decomposable torus or an abelian variety. Therefore $G$ is trivial or $G=\mathbb{Z}_{2}$ in Th. 4.6.

\section{ACKNOWLEDGEMEnTS}

We are grateful to the Tata Institute of Fundamental research in Mumbai, India, where this paper was conceived, and especially to I. Biswas and A.J. Parameswaran, organizers of our stay there.

\section{REFERENCES}

Bea83 A. Beauville, Variétés Kähleriennes dont la première classe de Chern est nulle, J. Differential Geom. 18 (1983), no. 4, 755-782.

BGL99 Ch. Birkenhake, V. González and H. Lange, Automorphism groups of 3-dimensional complex tori, J. reine angew. Math. 508 (1999), 99-125.

Bog74 F.A. Bogomolov, On the decomposition of Khler manifolds with trivial canonical class, Mat. USSR-Sb. 22 (1974), no. 4, 580-583.

Bru98 M. Brunella, Uniformisation of foliations by curves, in Holomorphic dynamical systems, Lecture Notes in Math., vol. 1998, Springer, Berlin, 2010, 105-163, arXiv:math/0802.4432.

Cam80 F. Campana, Réduction algébrique d'un morphisme faiblement Kählérien propre, Math. Ann. 256 (1981), 157-189. http://dx.doi.org/10.1007/BF01450796

Cam85 F. Campana, Réduction d'Albanese d'un morphisme propre et faiblement Kählérien et applications (I, II), Compositio Math. 54 (1985), 373-416.

Cam95 F. Campana, Fundamental group and positivity of cotangent bundles of compact Kähler manifolds, J. Algebraic Geom. 4 (1995), no. 3, 487-502.

Cam06 F. Campana, Isotrivialité de certaines familles Kählériennes de variétés non projectives, Math. Z. 252 (2006), 147-156, arXiv:math/0408148. http://dx.doi.org/10.1007/ s00209-005-0851-4

Dem91 J.-P. Demailly, Holomorphic Morse inequalities, in Several complex variables and complex geometry, Part 2 (Santa Cruz, CA, 1989), 93-114, Proc. Sympos. Pure Math., 52, Part 2, Amer. Math. Soc., Providence, RI, 1991. http://dx.doi.org/10.1090/pspum/052.2/1128538

Dem92 J.-P. Demailly, Regularization of closed positive currents and Intersection Theory, J. Algebraic Geom. 1 (1992), 361-409.

Dem07 J.-P. Demailly, Analytic methods in algebraic geometry, Lecture Notes, École d'été de Mathematiques de Grenoble "Géométrie des variétés projectives complexes : programme du modèle minimal" (June-July 2007). 


\section{F .Campana, J.-P. Demailly and M. Verbitsky}

DPa04 J.-P. Demailly and M. Paun, Numerical characterization of the Kähler cone of a compact Kähler manifold, Ann. of Math.(2) 159 (2004), 1247-1274, arXiv:math.AG/0105176. http://dx.doi. org/10.4007/annals. 2004.159.1247

DPe03 J.-P. Demailly and T. Peternell, A Kawamata-Viehweg Vanishing Theorem on compact Kähler manifolds, J. Differential Geom. 63 (2003), no. 2, 231-277. http://dx.doi.org/10.4310/SDG. 2003.v8.n1.a5

DPS01 J.-P. Demailly, T. Peternell and M. Schneider, Pseudo-effective line bundles on compact Kähler manifolds, Internat. J. Math. 12 (2001), 689-741. http://dx.doi.org/10.1142/ S0129167X01000861

Eno93 I. Enoki, Strong-Lefshetz-type theorem for semi-positive line bundles over compact Kähler manifolds, Geometry and global analysis (Sendai, 1993), 211-212, Tohoku Univ., Sendai, 1993.

Fle87 A.R. Fletcher Contributions to Riemann-Roch on projective threefolds with only canonical singularities Proc. Sympos. Pure Math. 46 (1987), 221-231. http://dx.doi.org/10.1090/pspum/ $046.1 / 927958$

Fuj83 A. Fujiki, On the structure of compact complex manifolds in $C$, in Algebraic varieties and analytic varieties (Tokyo, 1981), 231-302, Adv. Stud. Pure Math., 1, North-Holland, Amsterdam, 1983. http://dx.doi.org/10.2977/prims/1195175329

HP13 A. Höring and T. Peternell, Minimal Models for Kähler Threefolds, arXiv:1304.4013 (2013).

HPR11 A. Höring, T. Peternell and I. Radloff, Uniformisation in dimension four: toward a conjecture of Iitaka, arXiv:1103.539 (2011).

KV98 D. Kaledin and M. Verbitsky, Partial resolutions of Hilbert type, Dynkin diagrams, and generalized Kummer varieties, arXiv:math/9812078 (1998).

KV01 D. Kaledin and M. Verbitsky, Hyperholomorphic sheaves and new examples of hyperkähler manifolds, a chapter in the book Hyperkähler manifolds, International Press, Boston, 2001, arXiv:alggeom/9712012.

KM98 J. Kollár and S. Mori, Birational Geometry of Algebraic Varieties Cambridge Tracts in Mathematics 134, Cambridge University Press, 1998.

MMT08 R. Moosa, R. Moraru and M. Toma, An essentially saturated surface not of Kaehler type, Bull. Lond. Math. Soc. 40 (2008), no. 5, 845-854. http://dx.doi.org/10.1112/blms/bdn063

Moo05 R. Moosa, The model theory of compact complex spaces, Logic Colloquium 2001, Lect. Notes Log. 20, 317-349, Assoc. Symbol. Logic, 2005.

Mou99 C. Mourougane, Théorèmes d'annulation génériques pour les fibrés vectoriels semi-négatifs, Bull. Soc. Math. France 127 (1999), 115-133.

MP08 R. Moosa and A. Pillay, Model theory and Kaehler geometry, in Model Theory with Applications to Algebra and Analysis, Vol. 1, eds Z. Chatzidakis, D. Macpherson, A. Pillay and A. Wilkie, London Math. Soc. Lecture Note Ser. 349, Cambridge University Press, 2008, 167-195.

Pet98 T. Peternell, Towards a Mori theory on compact Kähler 3-folds, II. Math. Ann. 311 (1998), 729-764. http://dx.doi.org/10.1007/s002080050207

Pet01 T. Peternell, Towards a Mori theory on compact Kähler 3-folds, III. Bull. Soc. Math. France. 129 (2001), 339-356.

Siu74 Y.T. Siu, Analyticity of sets associated to Lelong numbers and the extension of closed positive currents, Invent. Math. 27 (1974), 53-156.

Siu84 Y.T. Siu, A vanishing theorem for semi-positive line bundles over non-Kähler manifolds. J. Differential Geom. 19 (1984), 431-452.

Tak97 K. Takegoshi, On cohomology groups of nef line bundles tensorized with multiplier ideal sheaves on compact Kähler manifolds, Osaka J. Math. 34 (1997), 783-802.

Uen75 K. Ueno, Classification theory of compact analytic spaces and algebraic varieties, Lecture Notes in Math., vol. 439, Springer, 1975. 


\section{KÄHLER THREEFOLDS HAVING NO SUBVARIETIES}

Ver98 M. Verbitsky, Trianalytic subvarieties of the Hilbert scheme of points on a K3 surface, Geom. Funct. Anal. 8 (1998), no. 4,732-782. http://dx.doi.org/10.1007/s000390050072

Ver04 M. Verbitsky, Coherent sheaves on generic compact tori, CRM Proc. Lecture Notes 38 (2004), 229-249.

Ver08 M. Verbitsky, Coherent sheaves on general K3 surfaces and tori, Pure Appl. Math. Q. 4 (2008), no. 3, part 2, 651-714, arXiv:math/0205210. http://dx.doi.org/10.4310/PAMQ.2008.v4.n3. a3

Ver09 M. Verbitsky, Parabolic nef currents on hyperkaehler manifolds, arXiv:0907.4217 (2009).

Frédéric Campana frederic.campana@univ-lorraine.fr

Institut Elie Cartan, Université Henri Poincaré, BP 239, 54506 Vandoeuvre-les-Nancy Cédex, France

and

Institut Universitaire de France

Jean-Pierre Demailly jean-pierre.demailly@ujf-grenoble.fr

Académie des Sciences

and

Université de Grenoble I, Institut Fourier, Laboratoire de Mathématiques, UMR 5582 du CNRS, BP 74, 100 rue des Maths, 38402 Saint-Martin d'Hères, France

Misha Verbitsky verbit@maths.gla.ac.uk, verbit@mccme.ru

Laboratory of Algebraic Geometry, SU-HSE, 7 Vavilova Str., Moscow 117312, Russia 